\title{
Dynamic mechanical properties of sugar palm/glass fiber reinforced thermoplastic polyurethane hybrid composites
}

\begin{abstract}
Hybrid composites based on thermoplastic polyurethane were fabricated with the addition of sugar palm fiber and glass fiber. The best ratio of $30 \mathrm{wt} \%$ sugar palm fiber to $10 \mathrm{wt} \%$ glass fiber was used to reinforce the hybrid composites, which were then subjected to dynamic mechanical analysis (DMA) to determine their dynamic properties. Three types of fiber treatment were assessed as follows: $6 \%$ alkaline (TNSP), 2\% silane (TSSP) and combined 6\% alkaline- 2\% silane (TNSSP) for $3 \mathrm{~h}$. The untreated and treated hybrid composites were fabricated by the meltcompounding method, followed by a hot pressing moulding. The DMA properties, including storage modulus, loss modulus and damping factor, were measured by a Dynamic Mechanical Analyzer. The hybrid composites of TNSSP showed highest storage modulus, loss modulus as compared to other treated hybrid composites. The peak height of damping factor was low for TNSSP and highest for TSSP hybrid composites. The Cole-Cole analysis was carried out to investigate the phase behavior of the untreated and treated hybrid composites. The study demonstrated that the combined $6 \%$ alkaline-2\% silane fiber treatment was the most effective in enhancing the dynamic mechanical properties of the SPF/GF reinforced TPU hybrid composites. It is clear that, the treated fibers influenced the DMA of thermoplastic polyurethane hybrid composites. The overall result showed that treated sugar pam/glass reinforced thermoplastic polyurethane improved the dynamic mechanical analysis for hybrid composites suitable for automotive parts.
\end{abstract}


